\title{
LA TERMINOLOGIA MARINARESCA STUDIATA DAL PUNTO DI VISTA DELLA FORMAZIONE DELLE PAROLE
}

Il presente articolo tratta della terminologia marinaresca. Si prendono in esame esempi di terminologia marinaresca nell'italiano d'oggi. É un settore della linguistica molto interessante dal punto di vista della formazione delle parole. I procedimenti formativi e $i$ meccanismi che regolano la formazione delle parole nell 'italiano d'oggi si studiano su questo corpus molto interessante e ricco: la formazione dei nomi riguarda la suffissazione, la prefissazione e in particolare la composizione, la formazione dei verbi riguarda la suffissazione, la prefissazione e soprattutto la formazione parasintetica che è propria dei verbi, mentre la formazione degli aggettivi, anche se presenta esempi di suffissati, di prefissati e di composti, è meno sviluppata.

\section{Introduzione}

Nel presente lavoro si prendono in esame esempi di terminologia marinaresca; termini relativi alla navigazione, alla vita sul mare e nella regione costiera. Le parole si studiano dal punto di vista della formazione delle parole. I procedimenti formativi e i meccanismi che regolano la formazione delle parole nell'italiano d'oggi si studiano su questo corpus molto caratteristico e ricco. L'approccio allo studio della formazione delle parole è sincronico. Si prendono in considerazione solo le parole motivate nella coscienza linguistica dei parlanti d'oggi.

La formazione delle parole comprende tre parti fondamentali: la suffissazione o la formazione delle parole con suffissi o la derivazione, la prefissazione o la formazione delle parole con prefissi e la composizione delle parole. I risultati di questi tre procedimenti formativi sono rispettivamente il suffissato o il derivato, il prefissato e il composto o la parola composta.

\section{Nomi formati}

\subsection{La suffissazione - I suffissati - I suffissati denominali}

La suffissazione comprende le formazioni tratte da diverse basi: nominali, aggettivali e verbali; questa tripartizione è fatta prendendo in considerazione il punto di partenza del procedimento formativo, cioè considerando la base della formazione ottenuta: si hanno i suffissati denominali, i suffissati deaggettivali e quelli deverbali. Prendendo in considerazione il punto d'arrivo del procedimento formativo, cioè la categoria d'arrivo, si hanno tre tipi formativi ancora: i suffissati nominali, i suffissati aggettivali e quelli verbali.

La base dei nomi derivati alberaggio 'tassa proporzionale alla qualità e alla quantità delle merci', alberata 'alberatura di una nave' e alberatura 'insieme degli alberi di 
una nave' è costituita dal nome albero, cioè 'albero della nave'; quindi si ha un microsistema a quattro termini: albero 'jarbol', alberaggio 'lučka pristojba', alberata e alberatura 'jarboli, katarke'.

La base dei nomi derivati ancoraggio, ancoressa, ancoretta e ancorotto è costituita dal nome ancora; quindi si ha un microsistema a cinque termini. Dalla base ancora si ha il nome ancoraggio formato con il suffisso -aggio; il nome indica l'azione di ancorare o di ancorarsi 'sidrenje, usidrenje', lo specchio d'acqua in cui le navi possono ormeggiare 'sidrište, kotvište' e la somma pagata da una nave per ormeggiare il natante in porto 'lučka pristojba'. Dalla stessa base si hanno i suffissati ancoressa 'grossa ancora', ancoretta e ancorotto, formati con i suffissi -essa, -etta e -otto; i nomi ancoretta e ancorotto oltre il significato diminutivo hanno un significato particolare: ancoretta 'amo per pesche speciali, a tre o quattro punte' e ancorotto 'ancora leggera usata da imbarcazioni di piccole dimensioni'.

La base dei nomi derivati baleniera, baleniere e balenotto è costituita dal nome balena; quindi dalla stessa base si hanno il nome baleniera, formato con il suffisso iera, che indica la nave attrezzata per la caccia alle balene 'kitolovac' e anche un tipo speciale di imbarcazione a remi che si usava all'epoca della navigazione a vela, il nome d'agente baleniere, formato con -iere, che indica il cacciatore di balene e il marinaio di una baleniera 'kitolovac' e il nome balenotto 'balena giovane', formato con -otto.

Il nome d'agente barcaiolo, che deriva da barca, indica chi usa o conduce una barca 'traghettatore, noleggiatore di barche'. I suffissati bastiere e bittone indicano oggetti: bastiere 'barra che mantiene le estremità inferiori delle sartie alla distanza stabilita'; bittone, accrescitivo in -otto che deriva dal nome bitta, in bittone d'ormeggio significa 'grossa bitta piantata sulla banchina'. La base dei suffissati bordame e bordata è costituita dal nome bordo e i suffissi sono -ame in bordame 'lato inferiore della vela' e -ata in bordata 'percorso a zig-zag che un natante compie per rimontare il vento'. Il suffissato cabinato, 'imbarcazione da diporto fornita di cabina', che deriva dal nome cabina, può essere a motore o a vela: cabinato a motore, cabinato a vela.

Si ha il suffisso -etto in calamaretto, derivato dal nome calamaro, che indica il calamare giovane, di piccole dimensioni, ricercato per le sue carni ottime, usate per fritture; e anche in gamberetto, suffissato derivato dal nome gambero, che indica vari crostacei di piccole dimensioni, per lo più marini, e commestibili. La base dei nomi fiocinata 'colpo di fiocina' e flocinatore 'pescatore esperto nel lanciare la fiocina' è costituita dal nome fiocina; dalla stessa base si ha un suffissato in -ata e un suffissato in -tore; il suffisso -tore è proprio dei nomi d'agente.

Il nome gommone 'cannotto pneumatico a remi o a motore fuoribordo' deriva dal nome gomma e il suffisso -one perde il suo valore accrescitivo; la gottazza 'grossa cucchiaia di legno per sgottare le imbarcazioni' deriva dal gotto, acquista il valore accrescitivo 'grosso, grande'. 
Il suffissato maretta è un diminutivo; deriva dal nome mare e indica una leggera agitazione del mare provocata dal vento. La base del suffissato marinaio è costituita dal nome marina; il suffissato marinaio è un nome d'agente 'persona che svolge la propria attività a bordo di una nave'; e il suffissato prodiere 'marinaio che svolge le sue mansioni a prua di un natante' è formato con il suffisso -iere aggiunto alla base costituita dal nome proda.

Anche il nome ostricaio, formato con il suffisso -aio', è un nome d'agente 'chi vende ostriche', 'venditore di ostriche', ma indica anche il luogo in cui si allevano ostriche.

I derivati che seguono si riferiscono al mare, alla pesca e al pesce. La base del nome derivato agugliara ' 'rete per la pesca delle aguglie' è costituita dal nome aguglia; alla base è aggiunto il suffisso - ara. Lo stesso suffisso -ara si ha in palamitara 'grande rete per la cattura di palamite' che deriva dal nome palamita e in polpara ${ }^{3}$ 'attrezzo per la pesca dei polpi' che deriva dal nome polpo. La base del nome pesciera 'vassoio per servire in tavola il pesce' è costituita dal nome pesce. Anche il suffissato retata, che deriva dal nome rete, è strettamente legato al mare e alla pesca e, come molti altri nomi formati con il suffisso - ata, indica gettata, quantità: 'gettata di rete' e 'quantità di pesce che si prende in una sola gettata'.

Il suffissato sartiame deriva dal nome sartia e il suffisso -ame aggiunge al nome un significato collettivo 'insieme delle sartie'. Anche il suffissato scogliera, che deriva dal nome scoglio, ha un significato collettivo, più precisamente "serie di scogli vicini tra loro'.

Il suffissato terzarolo ${ }^{4}$ deriva dal nome della vela, terzo, terza vela 'ripiegatura che si fa ad ogni vela per diminuire la superficie quando il vento soverchia'; la base dei suffissati timoneria 'insieme degli organi che azionano un timone', e timoniera 'locale di una nave dove si trovano il timone e gli altri organi di comando' è costituita dal nome timone; i suffissi sono -eria in timoneria e -iera in timoniera.

Dalla base tonno si ha il suffissato tonnara 'tunolovac' che indica l'impianto fisso con reti per la pesca dei tonni; il suffissato tonnarotto ${ }^{5}$ 'pescatore delle tonnare' è formato con il suffisso -otto; la base di tonnarotto è costituita dal derivato tonnara. Da tonno si ha il suffissato tonnetto, formato con -etto; oltre il significato diminutivo il suffissato contiene anche il concetto di somiglianza: tonnetto 'pesce di mare simile al

I Il nome ostricaro è un regionalismo. La base del suffissato è costituita dal nome ostrica. Il suffissato è formato con il suffisso -aro. È suffisso tipico di termini centro-meridionali e in particolare di termini marinareschi.

2 Il nome derivato agugliara è un regionalismo. Il suffisso -ara serve per formare i suffissati che indicano reti per catturare pesci o altri attrezzi usati per la pesca. I suffissati in -ara sono tipici dell'Italia meridionale.

3 Il suffissato polpara è un regionalismo che si usa nell' 'Italia meridionale.

4 Il nome trcarol (anche trcarul) 'skračena jedra, podveza za jedro' è un regionalismo. Dal nome trcarol si ha il verbo trcarolati 'uvijati jedra' che è anche un regionalismo. Il verbo italiano terzarolare, che è un verbo denominale, deriva dal nome terzarolo. Il nome trcarol e il verbo trcarolati sono italianismi entrati in croato.

5 Il suffissato tonnarotto è un regionalismo di origine siciliana. 
tonno, ma più piccolo del tonno'. Il suffissato totanara che deriva dal nome totano è formato con -ara; indica l'attrezzo per la pesca dei totani.

Alcuni derivati denominali che seguono si riferiscono alle navi e ai velieri; dalla base vapore si ha il suffissato vaporetto formato con -etto che aggiunge al suffisato il valore diminutivo 'piccola nave a vapore' e anche 'motonave di piccole dimensioni'; il suffissato trinchettina ${ }^{6}$, diminutivo di trinchetto da cui deriva, è la vela prodiera più bassa di un veliero con più fiocchi; il suffissato velaccino, diminutivo di velaccio da cui deriva ${ }^{7}$, è la seconda vela dall'alto dell'albero di trinchetto dei bastimenti a vele quadre; e il suffissato velacciere indica il veliero a tre alberi, di cui il prodiero a vele quadre e gli altri a vele latine.

\subsection{I suffissati deaggettivali}

I nomi derivati da aggettivi sono poco numerosi. I suffissati lunata e mezzana indicano vele; il suffissato lunata 'curva del lembo inferiore delle vele quadre' deriva dall'aggettivo lunato e il suffissato mezzana o albero di mezzana 'vela dell'albero più vicino alla poppa dei velieri a tre o a due alberi' deriva dall'aggettivo mezzano.

Dall'aggettivo marino si ha il nome marina che indica la superficie marina e la regione vicino al mare; dall'aggettivo derivato motonautico si ha il suffissato motonautica che indica la navigazione con imbarcazioni a motore; e dall'aggettivo composto navalmeccanico si ha il suffissato navalmeccanica che indica la scienza o la tecnica delle costruzioni meccaniche navali.

\subsection{I suffissati deverbali}

I sostantivi derivati da basi verbali sono numerosi. Prevalgono i deverbali a suffisso zero: afforco deriva dal verbo afforcare e indica l'ormeggio di una nave mediante due ancore di prua disposte ad angolo; approdo, che deriva dal verbo approdare, indica l'approdare o l'essere approdato, ma anche il luogo dove si può approdare; attrac$c o$, che deriva da attraccare, indica la manovra per attraccare e il luogo in cui si attraca; ormeggio, che deriva da ormeggiare indica l'ormeggiare, l'ormeggiarsi, l'essere ormeggiato, ma anche il posto in cui si ormeggia. Il deverbale a suffisso zero sbarco, che deriva da sbarcare, indica l'azione dello sbarcare e l'essere sbarcato, ma anche l'abbandono della vita di mare alla fine della carriera.

6 La base del suffissato trinchettina è costituita dal nome trinchetto di etimologia incerta. I nomi trinket "malo jedro na prednjem jarbolu', il nome trinketina 'trokutasto pomoćno jedro ispod prvog jarbola' sono italianismi accolti in croato.

7

8

nome velaccio proviene dallo spagnolo velacho che deriva dal nome vela 'vela'

La base del nome alaggio è costituita dal verbo alare 'tirare un cavo, una gomena'. Il verbo alare deriva dal verbo francese haler che è di origine germanica.

${ }^{9}$ La base del nome calafataggio è costituita dal verbo calafatare che deriva a sua volta dal nome calafato. Il nome kalafatanje e il verbo kalafatati ‘šuperiti, zatvarati pukotine između dijelova drvene građe' sono italianismi entrati in croato. 


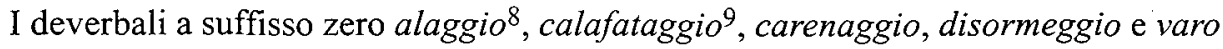
indicano l'operazione del verbo che costituisce la base del derivato: alaggio 'operazione del trarre in secco, su apposito scalo, un'imbarcazione', calafataggio 'operazione del calafatare', carenaggio 'operazione del carenaggio', disormeggio 'operazione del disormeggiare', varo 'operazione del varare, operazione che conclude la costruzione di una nave'.

Il suffissato affondamento 'affondare' formato con il suffisso -mento e il suffissato affondatoio 'congegno di leve per liberare l'ancora e lasciarla affondare' formato con il suffisso -toio derivano dal verbo affondare.

Il suffissato appoppamento 'inclinazione della nave verso poppa' deriva da appoppare/appopparsi e il suffissato appruamento 'inclinazione della nave verso prua' deriva da appruare/appruarsi; il derivato a suffisso zero beccheggio 'il beccheggiare', che deriva dal verbo beccheggiare, indica l'oscillazione della nave da poppa a prua.

Dal verbo italiano bordeggiare 'bordižati', 'kosati', che a sua volta deriva dal nome bordo, si hanno i suffissati bordeggiamento 'atto/effetto del bordeggiare', bordeggiata 'il bordeggiare' e 'spazio che una nave percorre bordeggiando' e bordeggio 'bordižanje' 'il bordeggiare', 'l'andatura per diagonali, con vento obliquo, cambiando di volta in volta di bordo' 10 .

I nomi impoppata 'forte spinta di vento da poppa', che deriva da impoppare, e mareggiata 'burrasca di mare e di vento', che deriva da mareggiare ${ }^{11}$, sono formati con il suffisso -ata; la base di orzata è costituita dal verbo orzare (o dal nome orza) e indica lo spostamento della prora verso la direzione da cui proviene il vento; poggia$t a$, che deriva da poggiare indica l'allontanare la prora dalla direzione onde spira il vento'; e remata, che deriva da remare, ha due significati: 'il remare a lungo, per un certo tempo' e 'colpo di remo nell'acqua e contro qualcosa o qualcuno'.

Dal verbo pescare transitivo si hanno i suffissati pesca, deverbale a suffisso zero 'il pescare' o 'ciò che si pesca', pescata 'il pescare' e 'quantità di pesce pescato in una volta', pescato 'quantità di pesce pescata in un determinato periodo di tempo', peschereccio 'grande motobarca attrezzata per la pesca', ribarica', pescheria 12 'mercato del pesce' o 'negozio dove si vende il pesce', peschiera 'vivaio di pesci'. Dal verbo pescare intransitivo si ha il nome pescaggio che indica l'altezza della parte di scafo che rimane immersa nell'acqua.

Dalla base rollare si hanno i nomi rollata 'ciascuna delle oscillazioni laterali della nave' e rollio 'movimento di oscillazione attorno al proprio asse longitudinale della nave'. Il nome scarroccio deriva da scarrocciare; è un deverbale a suffisso zero e indi-

10 Il verbo bordižati è un regionalismo; significa 'kosati', 'u jedrenju manevrirati ploveći protiv vjetra'. Il nome bordižanje è anche un regionalismo e significa 'manevriranje ploveći protiv vjetra'. Si usano nella regione costiera e nelle isole croate. Sono italianismi entrati in croato.

11 Il verbo mareggiare deriva a sua volta dal nome mare.

12 Il nome peškarija 'tržnica ribe, ribarnica' è un regionalismo che si usa nella regione costiera e nelle isole croate. 
ca lo spostamento laterale di una nave sotto l'azione del vento. Per quanto riguarda il lato semantico questi suffissati hanno in comune il concetto di movimento e di spostamento e dal punto di vista formativo sono microsistemi a tre termini.

Lo stazzamento 'lo stazzare', 'stazzatura', lo stazzatore 'operaio incaricato di stazzare le navi', e la stazzatura 'complesso delle operazioni per calcolare la stazza di una nave' derivano tutti e tre dal verbo stazzare; il deverbale a suffisso zero stiva che deriva da stivare ${ }^{13}$ indica gli spazi interni delle navi destinati ad accogliere il carico.

I nomi deverbali strambata, straorzata, veleggiata e vogata sono formati con il suffisso -ata: strambata 'rapido spostamento della randa da un bordo all'altro con il vento in poppa' deriva da strambare, straorzata 'spostamento violento della prua di una nave a vela verso la direzione dalla quale spira il vento' da straorzare, veleggiata 'il veleggiare' e 'breve gita in barca a vela' da veleggiare e vogata 'spinta con il remo per far avvanzare la barca' da vogare.

I nomi formati da basi verbali sono ancora: rematore 'chi rema', traghettatore 'chi traghetta' 'chi è addetto ai traghetti', vogatore 'chi voga', veleggiatore 'chi veleggia' e il nome indica anche un tipo di imbarcazione a vela.

\section{CAMPI SEMANTICI}

Classificazione dei nomi formati con suffissi secondo il loro significato:

nomi di oggetti/attrezzi/arnesi/impianti: affondatoio, afforco, agugliara, ancoressa, ancoretta, ancorotto, gottazza, palamitara, pesciera, polpara, rimorchio, sartiame, tonnara, totanara

nomi indicanti colpo/colpi di: fiocinata, retata, impoppata, mareggiata, remata nomi indicanti percorso/spostamento: bordata, bordeggiata, bordeggio, orzata, poggiata, rollata, rollio, scarroccio, strambata, straorzata, vogata

nomi collettivi: alberata, alberatura, sartiame

nomi d'agente (nomina agentis): baleniere, barcaiolo, fiocinatore, imbarcatore, marinaio, ostricaio/ostricaro, prodiere, sommergibilista, tonnarotto, rematore, rimorchiatore, stazzatore, traghettatore, veleggiatore, vogatore

nomi di animali/pesci: balenotto, calamaretto, gamberetto, tonnetto nomi di oggetti: allunamento, bastiere, bittone, boccalino, calderina, timoneria nomi di natanti: baleniera, cabinato, deriva, gommone, peschereccio, rimorchio, vaporetto, velacciere nomi di vele: bordame, lunata, mezzana, terzarolo, trinchettina, velaccio, velaccino

nomi indicanti quantità: retata, pescata, pescato

13 Il nome štiva 'potpalublje', 'prostor ispod palube' e il verbo štivati 'slagati teret u brodu', 'slagati teret u potpaljublju' sono regionalismi marinareschi. Il nome śtiva e il verbo štivati sono entrati in croato dal veneziano. Sono italianismi che si usano nella regione costiera croata e nelle isole, e in particolare in Dalmazia. 
nomi indicanti tassa: alberaggio, ancoraggio

nomi indicanti luogo: ancoraggio, attracco, scogliera, marina, imbarcatoio, ormeggio, pescheria, peschiera, stiva

nomi d'azione (nomina actionis): alaggio, ancoraggio, affondamento, afforco, appoppamento, appruamento, attracco, beccheggio, bordeggiamento, bordeggiata, bordeggio, calafataggio, carenaggio, dislocamento, disormeggio, ormeggio, pesca, pescata, rimorchio, sbarco, stazzamento, stazzatura, straorzata, varo, veleggiamento, veleggiata

nomi indicanti peso/immersione: tonnellaggio, dislocamento, pescaggio

nomi indicanti scienza: motonautica, navalmeccanica

nomi indicanti movimento/corrente: deriva, maretta

\subsection{La prefissazione - I prefissati}

La prefissazione o la formazione con prefissi occupa un posto importante nella formazione delle parole dell'italiano d'oggi. Ė un settore della formazione delle parole poco sviluppato nei nomi della terminologia marinaresca. I prefissi precedono la base e modificano semanticamente la base. La prefissazione non transcategorizza, non modifica la classe grammaticale, tranne nei parasintetici.

I nomi di alcune vele dei bastimenti a vele sono formati con il prefisso contro-: contromezzana 'la vela più bassa dell'albero di mezzana'; controvelaccino 'nei bastimenti a vele quadre la vela più alta dell'albero di trinchetto'; e controvelaccio 'anche nei bastimenti a vele quadre la vela più alta dell'albero di maestra'. Inoltre alcuni nomi che indicano elementi di costruzione delle navi sono formati con contro-: controchiglia, controelica, controfasciame.

Il prefissato entrobordo 'imbarcazione da turismo dotata di motore interno allo scafo' e 'il motore stesso' è costituito dal prefisso entro- e dal nome bordo; il prefissato fuoribordo 'imbarcazione con motore esterno allo scafo' e 'il motore stesso' è costituito dal prefisso fuori- e da bordo; in semicabinato 'imbarcazione da diporto con parte abitabile ridotta, generalmente a prua' il prefisso è semi-; e in sottomarino 'in grado di navigare in completa immersione' il prefisso è sotto-

\section{CAMPI SEMANTICI}

Classificazione dei nomi formati con prefissi secondo il loro significato:

elementi di costruzione: controchiglia, controelica, controfasciame nomi di natanti: entrobordo, fuoribordo, semicabinato, sottomarino nomi di vele: contromezzana, controvelaccino, controvelaccio parti di natanti: controcoperta, entrobordo, fuoribordo 


\subsection{La composizione - I composti}

La composizione delle parole unisce due o più elementi formativi, creando unità lessicali più grandi; questo procedimento formativo è caratteristico dell'italiano d'og$\mathrm{gi}^{14}$. Si prendono in esame $\mathrm{i}$ composti che sono motivati nella consapevolezza linguistica dei parlanti d'oggi; cioè il parlante continua a identificare gli elementi formativi dei composti anche dopo la fusione avvenuta: e il significato del composto è la somma dei significati degli elementi formativi. Per quanto riguarda i nomi composti nell'italiano d'oggi distinguiamo alcuni gruppi formativi che si hanno anche nella terminologia marinaresca: Nome + Nome: aliscafo, boccaporto, ecometro, motobarca; Aggettivo + Nome: l'aggettivo precede il nome che determina e specifica come in mezzavela, navalmeccanico (forme sintetiche), alta marea, bassa marea (forme analitiche); Verbo + Nome, modello formativo molto produttivo nell'italiano d'oggi: guardalato, parabordo; Preposizione + Nome: lungomare, lungotevere.

I composti Nome + Nome non sono numerosi. Il nome aliscafo 'hidrogliser' è costituito da ala e scafo e indica un'imbarcazione a motore provvista di ali che consentono allo scafo di sollevarsi sul pelo dell'acqua. Il nome boccaporto ${ }^{15}$ è costituito da bocca e porto e indica l'apertura/porta sul ponte delle navi che immette nei locali sottostanti e nelle stive. Anche il nome pescecane è costituito da due nomi.

I composti Aggettivo + Nome sono poco numerosi. Il nome mezzavela, costituito dall'aggettivo mezza e dal nome vela, indica ciascuna delle piccole vele che si distendono quando spira vento leggero da poppa. Mezzomarinaio, costituito dall'aggettivo mezzo e dal sostantivo marinaio, 'asta per l'attracco' e navalmeccanico 'operaio dell'industria navale' è costituito dall'aggettivo navale e dal sostantivo meccanico. In tutti e tre gli esempi l'aggettivo precede il nome. I composti Aggettivo + Nome alta marea e bassa marea sono forme analitiche.

Il modello formativo Verbo + Nome, molto produttivo nell'italiano d'oggi, è frequente nella terminologia marinaresca. Le basi verbali dei composti Verbo + Nome sono batti-, frangi-, guarda-, mostra-, para-, salva-: battipoppa 'pezza della tenda che pende a coprire le spalle di poppa', frangiflutti, frangimare e frangionde 'lukobran' indica la diga che protegge porti dalla violenza delle onde', guardacoste/guardiacoste 'battello adibito alla difesa delle coste e alla repressione del contrabbando', guardaporto 'nave posta all'ingresso di un porto militare con compiti di sorveglianza o di difesa', guardadighe 'chi è adetto alla sorveglianza di una diga', guardapesca 'chi è incaricato di far rispettare le leggi sulla pesca', guardalato e parabordo 'bokobran' indicano ripari appesi lungo i bordi di un natante per attutire gli urti', mostravento 'banderuola posta sull'albero di un veliero per indicare la direzione del vento', parabrezza 'nei motoscafi, vetro anteriore di protezione', paraonde 'riparo di lamiera,

14 Per questo problema si veda l'articolo Contributo allo studio della composizione delle parole. Raffronto contrastivo italiano-croato, croato-italiano. Primi risultati, Vučetić 1999.

15 Il nome bokaporta 'grotlo', 'ulaz u donji dio broda', 'otvor na palubi' è un italianismo entrato in croato. Si usa nella regione costiera croata e nelle isole. 
in coperta e attorno ai boccaporti, destinato a deviare lateralmente l'acqua accidentalmente imbarcata', salvagente 'oggetto galleggiante usato per imparare a nuotare o come mezzo di salvataggio. Il nome belvedere, costituito da bel(lo) e vedere indica il secondo pennone dall'alto e la relativa vela dell'albero di mezzana.

I composti Preposizione + Nome sono costituiti dalla preposizione lungo e da un nome: lungomare 'strada che costeggia la riva del mare', lungotevere 'strada che costeggia il Tevere'.

\subsection{La composizione allogena}

I composti con forma colta/con forme colte

I composti italiani con forma colta/con forme colte sono caratteristici dei linguaggi scientifici e tecnici e nascono con lo sviluppo della scienza, della tecnica e dell'industria. Le formazioni scientifiche e tecniche ottenute con elementi formativi di provenienza latina e greca o di provenienza moderna sono portatori di significato e formando una parola nuova mantengono il loro significato nella maggior parte dei casi ${ }^{16}$. Si tratta di un tipo particolare della composizione delle parole, molto diffuso nell'italiano d'oggi. Le formazioni del genere ottenute con elementi greci e latini o con elementi stranieri, prevalentemente inglesi e francesi, meritano di essere studiate nell'ambito della terminologia marinaresca soprattutto per la loro analiticità e per la loro produttività.

I sostantivi acquacoltura o acquicoltura, molluschicoltura o molluschicultura, ostricoltura, pescicoltura o piscicoltura, molluschicoltore o molluschicultore, pescicoltore o piscicoltore e ostricoltore si riferiscono all'allevamento sistematico di pesci o molluschi a scopo alimentare.

I nomi batimetria o batometria 'batimetrija', batigrafia 'batigrafija' sono costituiti da bati- 'profondo' e da -metria, -metro 'misura', 'misurazione' e -grafia 'scrittura', 'descrizione'; sono elementi formativi che si trovano in parole composte di formazione moderna. Batimetria o batometria e batigrafia indicano la parte dell'oceanografia che si occupa della misurazione delle profondità marine; e batimetro o batometro 'dubinomjer, batimetar' indica l'apparecchio per la misurazione delle profondità marine.

Il primo elemento eco- 'ambiente naturale' si trova in ecogoniometro 'apparecchio impiegato per la localizzazione di corpi immersi e la misurazione di distanze subacquee' e in ecometro e ecoscandaglio (o scandaglio acustico 'ekometar, morski dubinomjer'). Il nome idroscopio, formato da idro- 'acqua' e -scopio 'strumento impiegato per l'osservazione di determinati fenomeni', indica il cannocchiale per esplorare il fondo del mare. Il prefissoide idro- si trova in: idroplano 'hidroplan', costituito da idroe da (aero)plano 'un'imbarcazione con scafo generalmetne a fondo piatto', idroscivolante e idrovolante, costituiti da idro- e dal participio presente dei verbi scivolare e

16 Per questo problema si veda l'articolo sulla formazione allogena: Formazioni scientifiche. Primi materiali. Vučetić 1998. 
volare; sono imbarcazioni a fondo piatto in uso principalmente nelle regioni paludose. Il nome idroporto, costituito da idro(volante) e da (aero)porto, e il nome idroscalo, costituito da idro(volante) e da (aero)scalo, indicano aeroscali per idrovolanti.

Il prefissoide moto-, abbreviazione di motore, indica natanti azionati da motore a combustione interna in parole di formazione moderna: motobarca 'barca a motore', motonave 'nave mossa da uno o più motori', motopeschereccio 'grossa barca da pesca a motore', motoscafo 'imbarcazione veloce da diporto o da competizione', motovedetta 'nave piccola e veloce, adibita a servizio di polizia sul mare'.

Il composto multiscafo, costituito dal prefissoide multi- e da scafo e il composto poliscafo, costituito dal prefissoide poli- e da scafo sono imbarcazioni a più scafi.

I nomi radiobussola, radiofaro, radiogoniometro e radioscandaglio indicano apparecchi, strumenti di bordo che permettono a navi di definire la loro posizione geografica e di determinare la direzione di provenienza di segnali di un radiofono; si usano per esplorazioni subacquee. Sono costituiti da radio- che indica la relazione con onde elettromagnetiche e da un nome, semplice o formato: bussola, faro, goniometro, scandaglio.

Il composto autogonfiabile, costituito dal prefissoide auto- 'da sé, da solo' e dall'aggettivo gonfiabile, 'che si gonfia da sé, automaticamente' si usa come aggettivo e come sostantivo: canotto gonfiabile, zattera gonfiabile.

Il composto pescivendolo 'chi vende pesce', costituito dal nome pesce e dal suffissoide -vendolo 'venditore di' è una formazione moderna.

\section{CAMPI SEMANTICI:}

Classificazione dei nomi composti secondo il loro significato:

nomi di oggetti: guardalato, mezzomarinaio o mezzomarinaro, mostravento, parabordo, parabrezza, paraonde, salvagente

nomi di apparecchi per la navigazione: radiobussola, radiofaro, radiogoniometro

nomi di apparecchi che indicano misurazione e esplorazione delle profondità marine: batimetro o batometro, batiscafo, ecogoniometro, ecometro, ecoscandaglio, idroscopio

nomi di natanti: aliscafo, guardaporto, guardacoste, idroplano, idroscivolante, idrovolante, motobarca, motonave, motoscafo, motopeschereccio, motovedetta, multiscafo, poliscafo

parti di natanti: belvedere, boccaporto

nomi di luoghi/strade: idroporto, idroscalo, lungomare, lungotevere nomi d'agente: guardadighe, guardapesca, molluschicoltore, navalmeccanico, ostricoltore, pescicoltore, pescivendolo opere naturali/opere artificiali: frangiflutti, frangimare, frangionde nomi di vele: mezzavela, belvedere 
nomi indicanti allevamento di certe colture: acquacoltura o acquicoltura, molluschicoltura o molluschicultura, ostricoltura, pescicoltura

nomi indicanti scienza: batigrafia, batimetria o batometria

\subsection{Composti analitici}

Nell'italiano d'oggi si trova sempre più di frequente un tipo di composti molto caratteristico e economico che si trova anche nella terminologia marinaresca. Sono composti nominali, costituiti da soli nomi; il secondo nome determina il primo. Questo modello formativo è caratterizzato dall'assenza della preposizione davanti al secondo nome. La parola composta si ottiene per semplice accostamento di due nomi. Il secondo nome determina il primo, è in funzione di complemento di termine o di complemento di specificazione, oppure è in funzione di attributo: area giochi, alloggi equipaggio, alloggi ufficiali, antenna radio, cassa acqua potabile, galleria asse di trasmissione, sala comandi, sala macchine, serbatoio nafta.

\section{Aggettivi formati}

\subsection{La suffissazione - I suffissati - Gli aggettivi denominali}

Gli aggettivi batigrafico e batimetrico derivano dai composti batigrafia e batimetria e indicano che è relativo alla batigrafia o batimetria. La base dell'aggettivo motonautico 'che è relativo al motonauta' è costituita dal composto.

L'aggettivo marinaro che deriva dal nome marina, e l'aggettivo marinaresco, che deriva da marinaro, riguardano il mare, la navigazione e la vita sul mare; baleniero si riferisce alle balene e alla loro caccia e burrascoso 'che è in burrasca' è relativo al mare. Gli aggettivi arenoso, ciottoloso, ghiaioso, sabbioso, scoglioso, derivati da basi nominali, indicano pieno di, ricco di, coperto di, costituito da.

La base dell'aggettivo cabinato 'che è dotato di cabina' è costituita dal nome cabina. L'aggettivo orziero deriva dal nome orza 'lato di un'imbarcazione verso il quale soffia il vento' e si riferisce all'imbarcazione che tende a mettere la prora all'orza, cioè contro vento; la base del derivato prodiero 'di prua, del lato di prua' è costituita dal nome proda e si dice di nave che naviga anteriormente a un'altra, seguendo la medesima rotta. L'aggettivo timoniero 'del timone, che ha funzione di timone' deriva da $t i$ mone e l'aggettivo sommergibilista 'che appartiene all'equipaggio di un sommergibile' deriva da sommergibile.

\subsection{Gli aggettivi deverbali}

Gli aggettivi derivati da basi verbali sono rari: abbocchevole, navigabile, peschereccio, sommergibile. La base dell'aggettivo abbocchevole è costituita dal verbo $a b$ boccare; il derivato s'impiega per indicare un'imbarcazione che naviga inclinata su uno dei bordi, sfiorando il pelo dell'acqua. L'aggettivo navigabile deriva da navigare e indica la possibilità: si dice di specchio d'acqua in cui è posibile la navigazione. 
Anche l'aggettivo sommergibile, che deriva da sommergere, indica la possibilità 'che può sommergersi o immergersi'. L'aggettivo peschereccio, che deriva da pescare, si riferisce alla pesca.

\subsection{La prefissazione - I prefissati}

I nomi entrobordo e fuoribordo già menzionati si usano anche come aggettivi invariabili: motore entrobordo, imbarcazione entrobordo, motore fuoribordo, imbarcazione fuoribordo. Oltre all'aggettivo cabinato, già menzionato, si ha il prefissato semicabinato, usato come aggettivo. Il prefissato sottomarino 'che sta sotto la superficie del mare' si usa anche come aggettivo: cavo sottomarino, navigazione sottomarina, vegetazione sottomarina.

\subsection{La composizione - Gli aggettivi composti}

Gli aggettivi composti sono rari: aeronavale "si dice di attività o di operazione a cui partecipano aerei e navi'; autogonfiabile 'che si gonfia da sé, automaticamente'; navalmeccanico 'relativo alla meccanica navale'.

\section{CAMPI SEMANTICI:}

Classificazione degli aggettivi formati secondo il loro significato:

aggettivi di relazione: baleniero, batigrafico, batimetrico, burrascoso, entrobordo, fuoribordo, marinaro, marinaresco, metalmeccanico, motonautico, orziero, peschereccio, prodiero, sottomarino, timoniero

aggettivi di possibilità: abbocchevole, autogonfiabile, navigabile aggettivi indicanti essere dotato/ricco/pieno di/costituito da: arenoso, cabinato, ciottoloso, ghiaioso, roccioso, sabbioso, scoglioso, semicabinato aggettivi indicanti simile $a$ : arenoso, sabbioso aggettivi di appartenenza: sommergibilista aggettivi indicanti avere funzione di: timoniero

\section{Verbi formati}

\subsection{La suffissazione - I suffissati - I verbi denominali}

Il verbo intr. beccheggiare 'oscillare in senso longitudinale' deriva da becco e descrive il movimento longitudinale di un natante ${ }^{17}$. Il verbo intr. bordeggiare ${ }^{18}$ descrive il movimento del natante a vela che naviga a zig-zag per risalire il vento e il verbo tr.

\footnotetext{
17 Il verbo beccheggiare che deriva dal nome becco ricorda il movimento del becco dell'uccello che becca.

18 Abbiamo già parlato del verbo bordeggiare esaminando i suffissati derivati da basi verbali: bordeggiamento, bordeggiata, bordeggio derivano da bordeggiare.
} 
bordare 'spiegare e distendere una vela per farle prendere bene il vento' derivano tutti e due dal nome bordo. Dal nome albero della nave si ha il verbo tr. alberare 'munire di alberi una nave' e dal nome ancora si ha il verbo tr. ancorare 'ormeggiare un natante gettando l'ancora' e anche il verbo riflessivo ancorarsi 'ormeggiarsi, gettare l'ancora per ormeggiarsi'.

I verbi calafatare ${ }^{19}$, capponare e carenare sono transitivi e derivano tutti e tre da basi nominali: calafatare 'kalafatati', 'šuperiti', da calafato; capponare 'legare l'ancora al capo del cappone per sollevarla in coperta' da cappone ${ }^{20}$ e carenare 'mettere in secco la carena di una nave per le operazioni di manutenzione e di riparazione' da carena. In questo gruppo di verbi denominali solo il verbo intr. fiocinare si riferisce alla pesca: fiocinare 'lanciare la fiocina'; il verbo è transitivo quando significa 'colpire con la fiocina': fiocinare un pesce.

Il verbo intransitivo carteggiare, derivato da carta si riferisce alla navigazione marittima (o aerea) 'organizzare e verificare sulle carte una data rotta'. Il verbo transitivo costeggiare, che deriva da costa, può riferirsi al mare: costeggiare un'isola, un promontorio, una spiaggia, o usato assolutamente: la barca costeggia. La base del verbo intr. ondeggiare è costituita dal nome onda: muoversi a onde detto del mare, e oscillare seguendo il moto delle onde detto di imbarcazione.

I verbi denominali che seguono esprimono attività legate a imbarcazioni: il verbo tr. gottare 'togliere l'acqua dal fondo di un'imbarcazione', il verbo intr. orzare 'navigare con la prora contro vento', il verbo intr. poggiare 'allontanare la prora dell'imbarcazione a vela dalla direzione onde spira il vento', il verbo intr. remare 'vogare', il verbo tr. sartiare 'far scorrere la sartia attraverso un passaggio', il verbo tr. terzarolare $^{21}$ 'diminuire la superficie di una vela esposta al vento ripiegandola sui terzaroli', e il verbo intr. veleggiare 'navigare con un'imbarcazione a vela'. I verbi transitivi scandagliare e stazzare riguardano la misurazione: scandagliare 'misurare la profondità delle acque mediante lo scandaglio' e stazzare 'misurare la stazza di una nave o di un'imbarcazione a vela'.

\subsection{La prefissazione - I prefissati}

I prefissati sono pochi, formati con il prefisso dis- con significato negativo e di separazione 'liberare da': il verbo tr. disancorare 'liberare dall'ancora', il verbo rifl. disancorarsi 'liberarsi dall'ancora', il verbo tr. disincagliare 'liberare un natante con manovre di disincaglio', il verbo rifl. e intr. pron. disincagliarsi 'liberarsi con manovre di disincaglio' e il verbo tr. disormeggiare 'liberare dagli ormeggi, levare l'ormeggio'.

19 Abbiamo già parlato del verbo calafatare esaminando i suffissati derivati da basi verbali: calafataggio deriva da calafatare.

20 Il nome cappone o capone 'grosso paranco di prora usato per sollevare l'ancora' deriva dalla parola veneziana capon, accrescitivo del nome capo.

21 Abbiamo gia menzionato il nome terzarolo che deriva dal terzo, terza vela; dalla base terzo si ha il nome terzarolo, e da terzarolo si ha il verbo terzarolare. 


\subsection{I parasintetici/Le formazioni parasintetiche}

La formazione parasintetica è a cavallo tra suffissazione e prefissazione: il risultato di questo procedimento formativo è il parasintetico. Le formazioni parasintetiche sono caratteristiche dei verbi italiani formati da basi aggettivali e da quelle nominali. La formazione parasintetica consiste nell'aggiunta di un prefisso e di un suffisso sullo stesso livello formativo. Le formazioni del genere sono proprie dei verbi italiani.

Molti parasintetici sono formati con il prefisso $a$ - che esprime 'avvicinamento, direzione, addizione' ed ha funzione intensiva o derivativa: il verbo intr. abboccare all'amo 'afferrare l'esca con la bocca', e parlando di un natante a vela 'navigare inclinato con uno dei bordi che sfiora il pelo dell'acqua'; il verbo tr. accavigliare 'fissare i capi dei cavi alle caviglie', il verbo tr. accostare 'avvicinare il fianco di un'imbarcazione a quello di un'altra o a una struttura fissa', il verbo tr. affondare 'mandare a fondo', il verbo intr. pron. affondarsi 'andare a fondo, sprofondarsi', il verbo intr. pron. appopparsi 'essere troppo immerso verso poppa', il verbo intr. approdare 'toccare la proda', il verbo intr. appruare 'essere troppo immerso verso prua'. Il verbo dirottare è formato con il prefisso $d i$ - 'movimento'; il verbo tr. significa 'far deviare dalla rotta prevista una nave', e il verbo intr. significa 'cambiare rotta'. I verbi formati con il prefisso in-/im- 'dentro, sopra' sono: impoppare (tr.) 'far immergere un'imbarcazione più da prova che da prua', impopparsi (intr. pron.) 'essere troppo immerso verso poppa, appopparsi'; impruare (tr.) 'far immergere un'imbarcazione più da prua che da poppa' e impruarsi (intr. pron.) 'appruarsi'. Il verbo intr. sbandare è costituito dalla base banda 'ciascuno dei due lati di una nave', dal prefisso $s$ - e dal suffisso -are: 'inclinarsi su un fianco' detto di navi.

\section{CAMPI SEMANTICI:}

Classificazione dei verbi formati secondo il loro significato:

verbi esprimenti azione: alberare, ancorare, beccheggiare, bordare, bordeggiare, calafatare, capponare, carenare, carteggiare, costeggiare, dislocare, fiocinare, gottare, ormeggiare, orzare, poggiare, remare, sartiare, scandagliare, stazzare, terzarolare, spedare, sgottare, veleggiare

verbi esprimenti il trovarsi in uno stato: abbrumare, ancorarsi, appoppare e appopparsi, appruarsi, arenarsi, impopparsi, impruarsi, sbarcare, sbandare verbi esprimenti spostamento/percorso: accostare e accostarsi, affondare e affondarsi, approdare e approdarsi, bordeggiare, costeggiare, dirottare, dislocare, imbarcare e imbarcarsi, impoppare, impruare, incrociare, poggiare, remare, sbarcare, sbandare, veleggiare, vogare

verbi esprimenti misurazione: scandagliare, stazzare verbi indicanti il liberare da qualcosa: disancorare, disincagliare, disormeggiare 


\subsection{La composizione - I composti}

La composizione dei verbi è poco sviluppata. I verbi composti sono rari anche nella terminologia marinaresca; citiamo il verbo intransitivo straorzare costituito da stra- e orzare 'venire violentemente all'orza', 'volgere con violenza la prua verso la direzione dalla quale spira il vento'.

I verbi composti italiani, diffusi nella lingua d'oggi, sono formazioni analitiche, costituite da un verbo di significato largo e da un nome. Sono le cosiddette locuzioni verbali: fare acquisto, far uso....

I verbi composti italiani possono essere costituiti anche da un verbo e da un avverbio o da un verbo e da una preposizione: buttare fuori, buttare via, pensarci su; i verbi composti del genere non sono caratteristici della lingua italiana d'oggi e non sono propri della terminologia marinaresca.

\subsection{Composti analitici/Le locuzioni verbali $V+N$}

I composti analitici sono costituiti prevalentemente da un verbo e da un nome. Molti verbi italiani servono a formare i composti del genere; sono prevalentemente locuzioni verbali costituite da un verbo di significato largo, come andare, avere, dare, fare, mettere, prendere, tenere, e da un nome (o da qualche altro elemento ancora: preposizione). Molte locuzioni italiane equivalgono al verbo che è alla base del nome compreso nella locuzione, e più precisamente il nome della locuzione è in rapporto di derivazione con il verbo equivalente; i composti del genere sono caratteristici della terminologia marinaresca: andare all 'orza 'orzare', cambiar rotta 'dirottare', far terzarolo 'terzarolare', gettare l'ancora 'ancorare/ancorarsi', levare l'ancora, salpare l'ancora 'disancorare', levare gli ormeggi, sciogliere gli ormeggi 'disormeggiare', navigare a vela 'veleggiare'.

Si trovano più di frequente gli esempi di verbi composti analitici che non equivalgono al verbo che è alla base del nome compreso nel composto; sono composti costituiti da un verbo e da un nome, come amo, bordo, largo, mare, ormeggio, rotta, vela, vento (o da qualche altro elemento ancora: preposizione, altri nomi): gettare l'amo 'lanciare l'amo', girare di bordo, virare di bordo 'cambiare la rotta', prendere il largo 'partire', tenere il mare 'sopportare bene il mare mosso', gettare la rete 'lasciarla a mare per la pesca, gettare le reti 'lasciarle a mare per la pesca'.

Altri esempi: invertire la rotta 'mutare la rotta vera in rotta alla bussola', far rotta per un luogo 'esservi diretti, andarvi', seguire una rotta 'seguire il percorso lungo il quale procede una nave', tenere la rotta 'navigare mantenendo la rotta', alzare le vele, aprire le vele, bordare le vele 'spiegarle e partire', spiegare le vele 'partire', ammainare le vele 'far scendere alberetti, vele', far vela 'partire', 'uscire di porto', guadagnare il vento "navigare bordeggiando in modo da procedere verso la direzione da cui spira il vento', rimontare il vento, risalire il vento 'navigare contro vento', stringere il vento 'navigare disponendo le vele in modo che facciano il minor angolo possibile con la direzione dalla quale il vento spira', avere il vento in poppa 'avere il vento 
favorevole', navigare col vento in poppa 'navigare nella stessa direzione in cui il vento spira', dare le vele al vento 'navigare', mettere le vele al vento 'spiegarle e partire'.

\section{Avverbi formati}

\subsection{La prefissazione - I prefissati}

Gli avverbi formati sono pochi e sono formati con i prefissi contro-, sopra-, sottocontrocorrente 'contro corrente', sopracoperta stare sopraccoperta 'stare sul ponte di coperta', sopravvento 'dal lato da cui spira il vento', sottobordo sotto il bordo di una nave, accosto ad essa, sottocoperta 'sotto il ponte di coperta di una nave', sottovela 'con le vele bordate', sottovento 'dal lato opposto a quello da cui spira il vento'.

\section{Conclusione}

Nel presente articolo si prendono in esame esempi di terminologia marinaresca, termini relativi alla navigazione, alla vita sul mare e nelle regioni costiere. La suffissazione è propria dei nomi; sono frequenti i nomi derivati da basi nominali: ancoraggio, baleniera, ostricaio, retata; e in particolare i nomi derivati da basi verbali: appoppamento, appruamento, pescata, remata; nella formazione dei nomi derivati da basi verbali prevalgono i deverbali a suffisso zero: afforco, approdo, attracco, ormeggio. La prefissazione non è propria dei nomi. Nella formazione dei nomi prevale di gran lunga la composizione; il modello formativo produttivo nell'italiano d'oggi, il composto Verbo + Nome, è caratteristico della terminologia marinaresca: frangiflutti, guardalato, parabordo. I nomi composti formati con elementi formativi colti o formati modernamente sono molto frequenti nella terminologia marinaresca: batimetro, batiscafo, ecometro, ecoscandaglio, motobarca, motopeschereccio, poliscafo; le formazioni del genere sono caratteristiche dei linguaggi scientifici e tecnici. Si hanno anche i composti analitici nominali, costituiti da soli nomi, senza nessuna preposizione: alloggi equipaggio, sala comandi, sala macchine, serbatoio nafta.

Gli aggettivi formati non sono numerosi. Sono aggettivi di relazione, di possibilità, di somiglianza, di appartenenza e in particolare gli aggettivi indicanti dotato/ricco/pieno di e costituito da: marinaro, baleniero, prodiero, orziero, cabinato.

La formazione dei verbi riguarda la suffissazione, la prefissazione e la formazione parasintetica. I parasintetici sono propri della terminologia marinaresca: accostare, affondare, approdare, appruare, dirottare, impoppare, impruare, sbandare. I verbi composti veri e propri sono molto rari. Sono diffusi i composti analitici costituiti da un verbo di senso largo e da un nome: andare all'orza, aprire le vele, cambiar (la) rotta, dare le vele al vento, far terzarolo, gettare l'ancora, levare l'ancora, navigare a vela, spiegare le vele.

Nella formazione dei nomi e dei verbi troviamo alcuni italianismi entrati e accolti in croato: bokaporta, bordižanje, bordižati, kalafatanje, kalafatati, trcarol, trinketina. 


\section{Bibliografia}

Alinel, M. (1962), Dizionario inverso italiano, The Hague.

ANIĆ, V. (1998), Rječnik hrvatskoga jezika, Zagreb, Novi Liber.

ANIĆ, V. - Goldstein, I. (1999), Rječnik stranih riječi, Zagreb, Novi Liber.

BABIĆ, S.(1991), Tvorba riječi u hrvatskom književnom jeziku. Nacrt za gramatiku. HAZU, Zagreb, Globus.

BATTAGLIA, S. (a cura di) (dal 1961 in poi), Grande dizionario della lingua italiana, Torino, U.T.E.T.

DaRDANo, M. (1978), La formazione delle parole nell 'italiano di oggi, Roma, Bulzoni.

DARDANO, M. (1986), Il linguaggio dei giornali italiani, Bari, Laterza.

De Mauro, T. (1987), Storia linguistica dell'Italia unita, Bari, Laterza.

GarZANTI ed. (1998), Il Grande Dizionario Garzanti della lingua italiana, Milano.

Greimas, A. J. (1986), Sémantique structurale, Paris, PUF.

KLAIĆ, B. (1986) Rječnik stranih riječi. Tuđ̈ice i posuđenice, Zagreb, Nakladni Zavod MH.

LeumanN, M. (1944), Gruppierung und Funktionen der Wortbildungssuffixe des Lateins, in «Museum Helveticum» I, 3, Basel, pp. 129-151.

MarChand, H. (1969), The Categorie and Types of Present-Day English Word-Formation, München.

MARTINET, A. (1967), Éléments de linguistique générale, Paris, Armand Colin.

$\mathrm{MEDIC1}$, M. (1978), La lingua delle pagine gialle, Torino.

Medici, M. (1986), La parola pubblicitaria, Venezia, Marsilio.

Migliorini B. (1965), Vocabolario della lingua italiana (Edizione rinnovata del Vocabolario della lingua italiana di G. Cappuccini e B. Migliorini), Torino.

MiglorinI B. (1988), Storia della lingua italiana. Introduzione di G.Ghinassi), Torino.

Miglorini, B. - Baldelli, I. (1981), Breve storia della lingua italiana, Firenze, Sansoni.

PotTier, B. (1992), Sémantique générale, Paris, PUF.

Renzi, L. - Salvi, G. - CARDINALETti, A. (a cura di) (1995), Grande grammatica italiana di consultazione, vol. III, Tipi di frasi, deissi, formazione delle parole, Bologna, Il Mulino.

ROHLFS, G. (1954), Historische Grammatik der italienischen Sprache und ihrer Mundarten, Band III: Syntax und Wordbildung, Bern, A. Francke.

SCHIAfFINI, A. (1963-64), La formazione del lessico italiano, dispense universitarie, Roma.

Serianni, L. - Trifone, P. (a cura di) (1994), Storia della lingua italiana, Vol. Il, Scritto e parlato, Torino, Einaudi.

Tekavéı́c, P. (1972, 1980), Grammatica storica dell'italiano, Vol. III: Lessico, Bologna, Il Mulino.

TOLLEMACHE F. (1945), Le parole composte nella lingua italiana, Roma, Rores.

VIDOvić, R. (1984), Pomorski rječnik, Split, Logos.

VuĆETIĆ, Z. (1979), Suvremeni talijanski jezik. Tvorba riječi. (L'italiano contemporaneo. La formazione delle parole), Zagreb, Liber.

VuĆETIĆ, Z. (1998), Formazioni scientifiche. Primi materiali, in «Linguistica» XXXVIII,2, Ljubljana, pp. 167-182.

VUČETIĆ, Z. (1999), Contributo allo studio della composizione delle parole. Raffronto contrastivo italiano-croato, croato-italiano. Primi risultati, in «Linguisitca» XXXIX, Ljubljana, pp. 83-98.

Zingarelli, N. (1996), Vocabolario della lingua italiana, dodicesima edizione, Bologna, Zanichelli. 


\section{Povzetek}

\section{POMORSKA TERMINOLOGIJA S STALIŠČA BESEDNE TVORBE}

Prispevek se ukvarja s terminologijo pomorstva, življenja na morju in ob morju, in sicer $z$ vidika besedne tvorbe $\mathrm{v}$ italijanščini, navaja pa tudi termine, ki so kot italianizmi postali last hrvaškega jezika. tako npr. bokaporta, bordižanje, kalafetanje, kalafatati, trcarol, trinketina.

Ugotavlja se, da so pri samostalnikih najbolj pogostne izvedenke iz samostalniških in glagolskih osnov, manj iz pridevniških. Pri glagolskih izvedenkah jih je kar nekaj z ničto pripono. Preponske derivacija je šibko zaznavna pri samostalnikih, pač pa je pogostna tvorba glagol + samostalnik. Samostalnik je močno zatopan $v$ učenih tehničnih izrazih, sestavljenih iz grških in latinskih jezikovnih prvin. Za samostalniške tvorbe so značilne sestavljenke $z$ dvema samostalnikoma brez predloga.

Glagolska tvorba je ugotovljiva s pomočjo pripon in predpon, močno pa je zastopana tudi parasintetična tvorba. 